Imaging X-ray crystal spectrometer for laser-produced plasmas

This article has been downloaded from IOPscience. Please scroll down to see the full text article.

2011 JINST 6 P04004

(http://iopscience.iop.org/1748-0221/6/04/P04004)

View the table of contents for this issue, or go to the journal homepage for more

Download details:

IP Address: 141.211.173.82

The article was downloaded on 06/04/2012 at 16:52

Please note that terms and conditions apply. 


\title{
Imaging X-ray crystal spectrometer for laser-produced plasmas
}

\author{
E.J. Gamboa, ${ }^{a, 1}$ D.S. Montgomery, ${ }^{b}$ I.M. Hall ${ }^{c}$ and R P. Drake ${ }^{a}$ \\ ${ }^{a}$ University of Michigan, \\ 2455 Hayward St, Ann Arbor, MI 48109 U.S.A. \\ ${ }^{b}$ Los Alamos National Laboratory, \\ Los Alamos, NM 87545 U.S.A. \\ ${ }^{c}$ University of Nevada, Reno, \\ 1664 N Virginia St, Reno, NV 89557 U.S.A. \\ E-mail: eliseo@umich.edu
}

ABSTRACT: X-ray Thomson scattering (XRTS) is a powerful technique for measuring state variables in dense plasmas. In this paper, we report on the development of a one-dimensional imaging spectrometer for use in characterizing spatially nonuniform, dense plasmas using XRTS. Diffraction of scattered x-rays from a toroidally curved crystal images along a one-dimensional spatial profile while simultaneously spectrally resolving along the other. An imaging spectrometer was fielded at the Trident laser at Los Alamos National Laboratory, yielding a FWHM spatial resolution of $<25 \mu \mathrm{m}$, spectral resolution of $4 \mathrm{eV}$, spectral range of $350 \mathrm{eV}$, and spatial range of $>3 \mathrm{~mm}$. A geometrical analysis is performed yielding a simple analytical expression for the throughput of the imaging spectrometer scheme. The SHADOW code is used to perform a ray tracing analysis on the spectrometer fielded at the Trident Laser Facility understand the alignment tolerances on the spatial and spectral resolutions. The analytical expression for the throughput was found to agree well with the results from the ray tracing.

KEYWORDS: X-ray detectors; Plasma diagnostics - interferometry, spectroscopy and imaging

${ }^{1}$ Corresponding author 


\section{Contents}

1 Introduction 1

2 Theory 2

2.1 Geometrical definitions 2

2.2 Imaging spectrometer geometrical analysis $\quad 4$

$\begin{array}{lll}2.3 & \text { Crystal throughput } & 5\end{array}$

$\begin{array}{llr}2.4 & \text { Ray tracing analysis } & 6\end{array}$

3 Tests of the spatial and spectral resolution $\quad 6$

4 Conclusion $\quad 10$

\section{Introduction}

Experiments in the 1930s have demonstrated that the performance of Bragg crystal spectrometers could be dramatically improved by curving the crystal. With a cylindrically-curved crystal, the spectrometer has mitigated the effects of source broadening as in the Johann and Johansson schemes $[1,2]$ or has substantially increased the brightness of the spectrum while simultaneously spatially resolving the source as with the von Hámos scheme [3]. Improvement in crystal fabrication in later years made it possible to create $\mathrm{x}$-ray optics based on spherically-bent crystals. These could either be arranged to provide two-dimensional imaging within a narrow spectral window [4], or combining the imaging of the von Hámos spectrometer with the improved dispersion of the Johann scheme [5].

Because of the relative difficulty in aligning crystal optics for fine resolution, a common approach in high-energy density facilities is to use a flat or singly-curved crystal optic. Mosaic crystals, which consist of randomly aligned grains of crystals, such as highly-oriented pyrolytic graphite (HOPG) are commonly used for their high-brightness in mosaic focussing mode [6, 7]. However, the spatial and spectral resolution achievable in a von Hamos configuration using mosaic crystals is limited by aberrations in the crystal and the wide spectral bandpass [8].

The FSSR-1D scheme, employing a spherical crystal for one-dimensional spatial focusing and spectral dispersion, has been used extensively in high-energy density physics (HEDP) experiments [9-14]. This configuration uses relatively easily manufactured and aligned spherical crystals to achieve high spectral and spatial resolution when certain geometric constraints can be met. These constraints arise from the fact that the Bragg angle is limited to larger than 45 degrees [15] and must to be greater than 80 degrees to limit astigmatism [16].

Perhaps for this reason, spherical crystals have been used much less extensively in spectroscopic applications at high energy density. As we discuss further below, our focus here is on the need for a compact diagnostic that can be inserted and aligned from a single direction and within a 
relatively small solid angle. This is important in the context of high-energy-density facilities such as Omega [17] or the National Ignition Facility [18] in which diagnostic access and perhaps time for alignment are very limited.

Advances in optics technologies have allowed the mating of high quality crystals with toroidally curved substrates [19]. With unequal radii of curvature, toroidal crystals can be made free from astigmatism so that any Bragg angle may be chosen. This greatly increases the flexibility for the instrument to fit in tight geometries. These crystals have been used to create two-dimensional, monochromatic images with high resolution [19-21]. Here we show how such a crystal can be used to create an imaging spectrometer [22]. The Bragg angle may be made small, with the spatial resolution limited only by higher-order aberrations like coma, so that the instrument can be inserted and aligned from a single direction.

Since the experiments of Compton [23], observing scattered radiation has been a fundamental technique to measure the physical parameters of dense materials. In a high-energy density physics context, XRTS was first proposed as a method to diagnose solid-density plasmas by Landen et al. [24] and given an extensive theoretical description by Gregori et al. [25]. Subsequent experiments have used XRTS as a method to diagnose isochorically-heated plasmas [26-28] . XRTS has been applied to hydrodynamic plasma flows typically through defining a narrow scattering window and time-gating the spectrometer [29-32].

In the present work, we report on the development of an imaging spectrometer for use in diagnosing spatially inhomogenous plasmas via XRTS. A toroidally curved, perfect Ge crystal affords high spatial resolution within a large field-of-view combined with spectral resolution that is an order of magnitude higher than is available with more commonly used HOPG spectrometers. Using this instrument, the goal is to be able to use XRTS to simultaneously characterize a large volume region to see the evolution of plasmas in experiments in warm-dense matter, radiating shocks, and many more potential applications in high-energy density physics.

\section{Theory}

To better understand and optimize the choice of a crystal for a toroidal imaging spectrometer, we present a geometrical analysis of the system to calculate the brightness of the resulting image. This analysis is similar to a derivation presented by Missalla et al. [33]. The throughput of the image is defined in terms of the effective solid angle of the detector with respect to the source. Since the spectrometer has a wide spectral range, the calculation is carried out for a monochromatic source. The assumption is that the crystal is small so that the central energy approximately describes the complete energy range.

\subsection{Geometrical definitions}

A perfect Bragg crystal forms the basis of the diagnostic. X-ray photons of wavelength $\lambda$ are dispersed according to the well known Bragg law,

$$
\sin \theta_{B}=m \lambda / 2 d
$$

where $\theta_{B}$ is the Bragg angle, $m$ is the diffraction order, and $d$ is the spacing of the crystal lattice planes. The crystal is bent along a toroidal surface so that it has two different radii of curvature. 
The focal lengths of the horizontal and vertical curvatures are related to the two bending radii, $R_{h}$ and $R_{v}$, respectively, and the Bragg angle.

$$
f_{h}=\frac{1}{2} R_{h} \sin \theta_{B}, \quad f_{v}=\frac{R_{v}}{2 \sin \theta_{B}}
$$

For a single photon energy, the condition for stigmatic two-dimensional imaging may be met if $R_{h}$ and $R_{v}$ are chosen such that $f_{h}=f_{v}$. One-dimensional imaging within a spectral window is possible if the lensmaker's equation is satisfied for the vertical curvature only.

$$
\frac{1}{d_{s c}}+\frac{1}{d_{c d}}=\frac{1}{f_{v}}
$$

We set the crystal-to-detector distance, $d_{c d}$, such that the detector lies on the Rowland circle defined by the horizontal curvature

$$
d_{c d}=R_{h} \sin \theta_{B}
$$

Solving (2.3) for the source-to-crystal distance, $d_{s c}$, in terms of the magnification $M=d_{c d} / d_{s c}$ yields

$$
d_{s c}=\frac{R_{v}}{2 \sin \theta_{B}} \frac{M+1}{M}
$$

The crystal face is selected as a section of the convex/convex face of a three-dimensional toroid. The chosen coordinate system has the origin at the center of the toroid as shown in figure 1. The center of the crystal is defined as point $C$. An arbitrary point $P$ on the surface of the toroid is described by the vector $\overrightarrow{O P}$. This vector is a function of a horizontal angular displacement $\alpha$ and a vertical angle $\gamma$ as measured from the axis along vector $\overrightarrow{O C}$

Photons are emitted by a point source, at position $S$. The central source-to-crystal vector $\overrightarrow{S C}$ makes an angle $\theta_{B}$ with the center of the crystal face and has a magnitude $d_{s c}$. Likewise, the central crystal-to-detector vector $\overrightarrow{C D}$ has a magnitude of $d_{c d}$. It is assumed that a photon that hits the crystal at a point $P$ will specularly reflect with respect to the local crystal normal $\hat{n}_{\text {crys }}$.

We can write these terms as

$$
\begin{aligned}
\overrightarrow{O S} & =\left(R_{h}-d_{s c} \sin \theta_{B},-d_{s c} \cos \theta_{B}, 0\right) \\
\overrightarrow{O P} & =\left(\eta \cos \alpha, \eta \sin \alpha, R_{v} \sin \gamma\right) \\
\overrightarrow{O C} & =\left(R_{h}, 0,0\right) \\
\hat{n}_{c r y s} & =(-\cos \gamma \cos \alpha,-\cos \gamma \sin \alpha,-\sin \gamma) \\
\eta & =R_{h}+R_{v}(\cos \gamma-1)
\end{aligned}
$$

We are interested in calculating the variation of the Bragg angle in terms of coordinates with respect to the source, the horizontal and vertical angles $\left(\beta, \gamma^{\prime}\right)$. Bragg diffraction will occur as long as the angular deviation from the Bragg angle is within the non-zero region of the rocking curve function, which describes the angular acceptance of the crystal [34]. By calculating the deviation of the Bragg angle as a function of the displacement angles $\left(\beta, \gamma^{\prime}\right)$, we may express the active area in terms of a solid angle with respect to the source. Thus, this calculation will define the throughput of the crystal. 


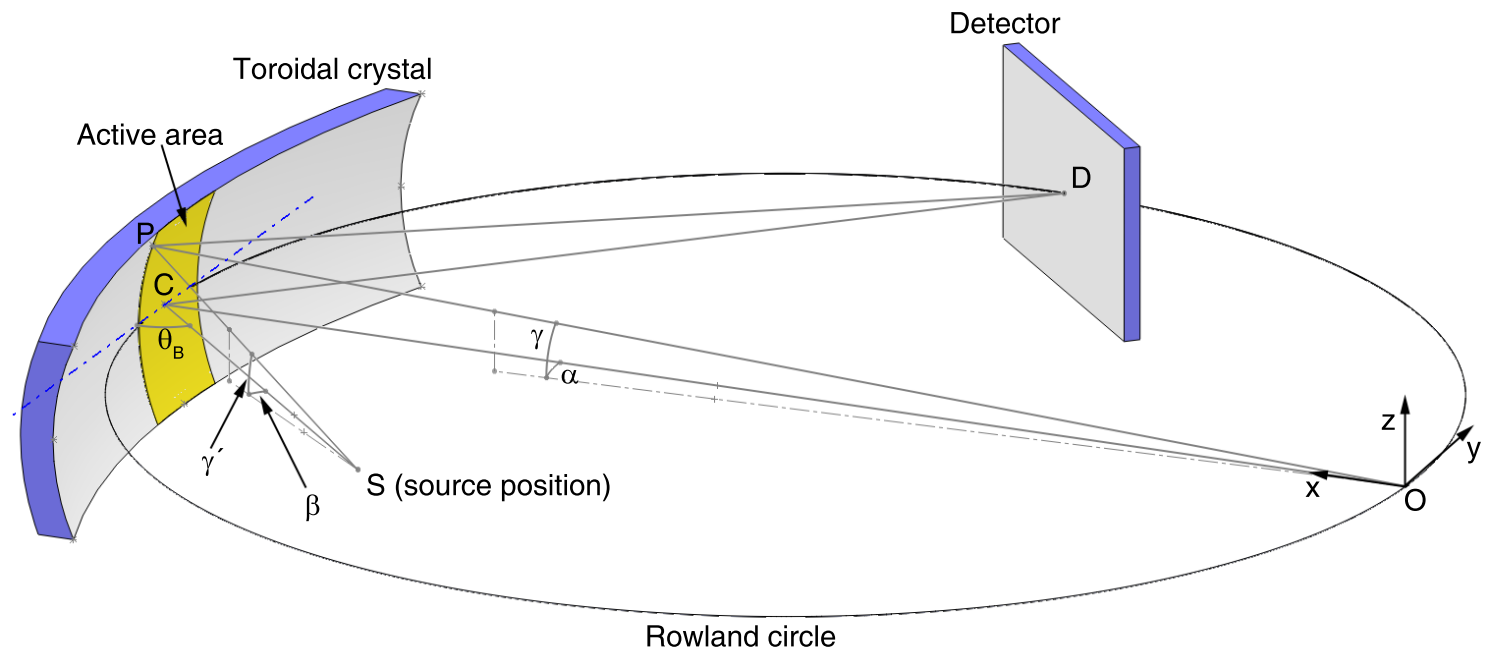

Figure 1. This sketch shows a definition of the geometry used in the analysis of toroidally curved imaging spectrometers. The horizontal curvature is defined along the xy-plane while the vertical curvature is along the xz-plane.

\subsection{Imaging spectrometer geometrical analysis}

For a ray that is displaced by a small angle $\left(\beta, \gamma^{\prime}\right)$ from the central vector $\overrightarrow{S C}$ to the point $P$, the angle that the ray makes with the local crystal normal is modified by a small increment $\delta$ from the Bragg angle. We can write the local angle of incidence $\theta^{\prime}$ as a function of the angular displacements and the central Bragg angle of incidence $\theta_{B}^{\prime}=\pi / 2-\theta_{B}$.

$$
\theta^{\prime}=\theta_{B}^{\prime}+\delta\left(\beta, \gamma^{\prime}\right)
$$

We are interested in the angle of incidence of the source-to-intersection vector, $\overrightarrow{S P}=\overrightarrow{O S}-\overrightarrow{O P}$, relative to the local crystal normal.

$$
\theta^{\prime}=\arccos \left(\frac{\overrightarrow{S P}}{|\overrightarrow{S P}|} \cdot \hat{n}_{\text {crys }}\right)
$$

We may compute this angle using (2.6)-(2.10). Applying the small angle approximation for $\alpha$ and $\gamma$ and taking terms up to first order yields

$$
\theta^{\prime}=\pi / 2-\theta_{B}+\left(\frac{R_{h} \sin \theta_{B}-d_{s c}}{d_{s c}}\right) \alpha
$$

We can transform the angular deviations back to source-coordinates via the relation

$$
\alpha=\beta+\theta_{B}^{\prime}-\theta^{\prime}
$$

Using (2.4), (2.5), (2.11), and (2.14), (2.13) can be simplified as

$$
\delta(\beta)=\left(\frac{M-1}{M}\right) \beta
$$


Up to first order, deviations in the Bragg angle are affected by only variations in the horizontal angle $\beta$. The physical meaning is that, for a given energy, the active area on the crystal forms a thin vertical strip.

Bending the crystal modifies the interaction with x-rays in two ways. The crystal lattice is modified on a microscopic scale, so that the angle that an incident ray makes with the distorted crystal planes changes as it moves through the surface layer of the crystal. This leads to an enhancement of the integrated reflectivity for bent crystals [35]. The second effect is apparent from (2.15). The magnification of the imaging spectrometer changes the geometrical variation of the incident angle with the crystal face.

In the absence of geometrical effects from the choice of mount, we denote the integrated reflectivity of a curved crystal by the term $R_{\text {int }}$. For simplicity, we assume that the rocking curve is described by a Gaussian function $R C(\delta(\beta))$. Substituting in eq. (2.15) yields the relationship

$$
R C(\delta(\beta))=R C\left(\left(\frac{M-1}{M}\right) \beta\right)
$$

The integrated reflectivity is defined as the integral of the rocking curve over all angles of incidence. Evaluating the integral, we recover the curved crystal integrated reflectivity modified by a geometrical term.

$$
R_{\text {int }}^{\prime}=R_{\text {int }}\left|\frac{M}{M-1}\right|
$$

\subsection{Crystal throughput}

The active area on the crystal is defined by a thin width $\Delta y_{m}$ in the horizontal (dispersion) direction and a length $\Delta x_{m}$ in the vertical (imaging) direction. The width of the active area is approximately the length over which the incident angle varies by the geometrically modified integrated reflectivity

$$
\Delta y_{m} \approx \frac{d_{s c} R_{\mathrm{int}}^{\prime}}{\sin \theta_{B}}
$$

The crystal focuses all radiation over its vertical dimension $S_{v}$ so that the length of the active area is $\Delta x_{m}=S_{v}$. The solid angle subtended by the active area with respect to the source is then

$$
\Omega_{a c t}=\frac{\Delta x_{m} \Delta y_{m} \sin \theta_{B}}{d_{s c}^{2}}=\frac{S_{v}}{d_{s c}} R_{\mathrm{int}}^{\prime}
$$

Substituting in eq. (2.5) and (2.17) into eq. (2.19) yields

$$
\Omega_{a c t}=2 \sin \theta_{B} R_{\text {int }}\left(\frac{S_{v}}{R_{v}}\right)\left|\frac{M^{2}}{M^{2}-1}\right|
$$

For the spectrometer described in the next section, this equation gives a throughput of $1.82 \times 10^{-5} \mathrm{sr}$.

While this value is an order of magnitude below the throughput for the more commonly used HOPG spectrometers [36], in certain types of experiments imaging spectrometers using perfect crystals can produce brighter images compared to their non-imaging HOPG counterparts. Using XRTS to diagnose hydrodynamic flows with a non-imaging crystal normally requires time gating the detector (typically using a microchannel plate that offers detection efficiencies of a few percent) and designing a narrow spatial window into the scattering volume. An imaging spectrometer may use an order of magnitude more sensitive image plate or deep-depletion CCD detector while capturing scattering along a large spatial chord in the scattering volume. 


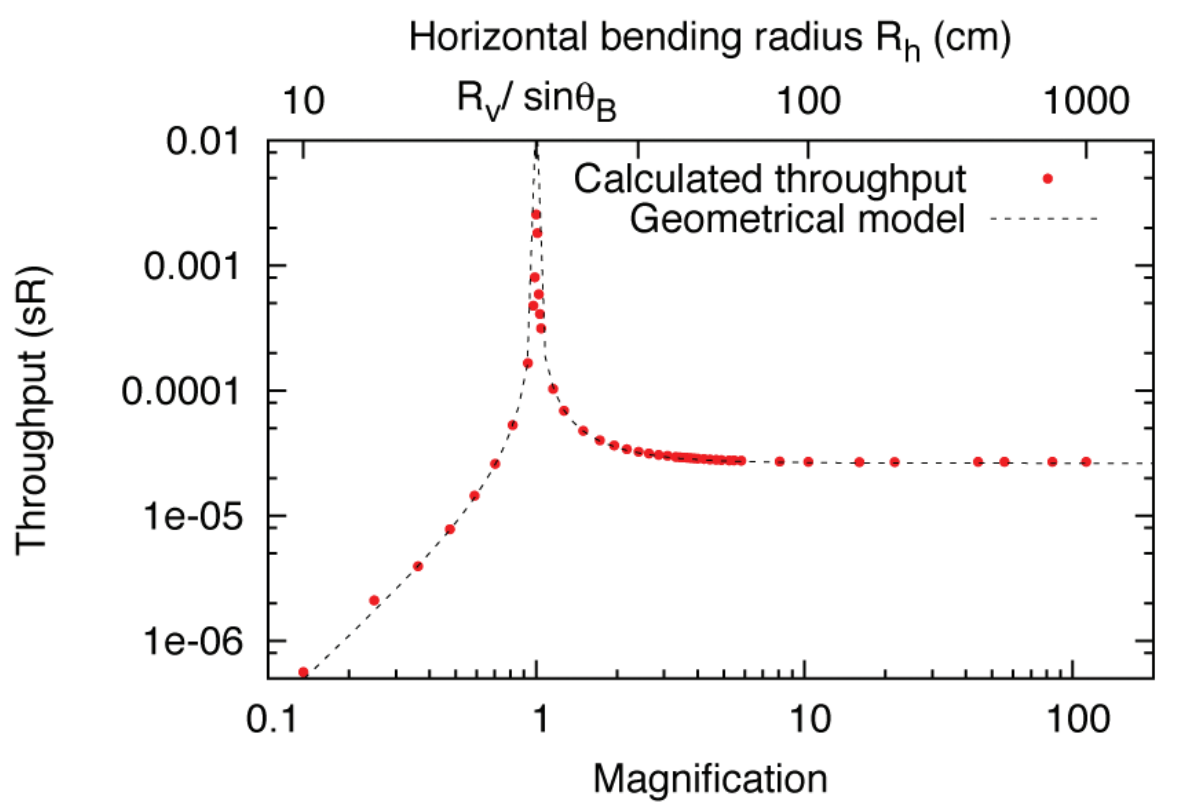

Figure 2. Plotted is the analytical expression for the throughput from (2.20) compared to the results from a ray tracing analysis.

\subsection{Ray tracing analysis}

Ray tracing calculations were carried out using SHADOW [37] for an instrument using a $\mathrm{Ge}(400)$ crystal with $R_{v}=15 \mathrm{~cm}$, a nominal $R_{h}=30 \mathrm{~cm}, S_{v}=2 \mathrm{~cm}$, and $R_{\text {int }}=0.107 \mathrm{mrad}$. Illumination was provided by a monochromatic point source at $4750 \mathrm{eV}$ and $\theta_{B}=67.33^{\circ} . R_{h}$ was varied from $f_{v}$ to $1000 \mathrm{~cm}$ with $d_{s c}$ adjusted by means of eq. (2.5) to fulfill the focusing condition. The throughput was calculated and plotted against the magnification as shown in figure 2. Also plotted is the analytical expression from (2.20).

The analytical expression agrees with the ray tracing results except in the limit of $M \rightarrow 1$. To describe the throughput at this position, it is necessary to take higher order terms in the expansion of eq. (2.13). A second order term $1 /\left(2 \tan \theta_{B}\right) \alpha^{2}$ describes the variation in the angle as the $(M-1) \alpha$ term goes to 0 . This limits to a finite value the asymptotic behavior at $M=1$.

\section{Tests of the spatial and spectral resolution}

To determine the spatial and spectral resolving characteristics of the described imaging spectrometer, a diagnostic was fielded at the 200-TW Trident Laser Facility at Los Alamos National Laboratory [38]. The spectrometer was fabricated using a Ge(400) crystal with $R_{v}=20 \mathrm{~cm}, R_{h}=40 \mathrm{~cm}$, $M=2.5$, and $\theta_{B}=70.25^{\circ}$ to observe the helium-like emission from a Ti source. The crystal length in the dispersion direction was $5 \mathrm{~cm}$, yielding a spectral range of $350 \mathrm{eV}$.

Shots were performed on a series of grid targets to evaluate the resolution of the spectrometer. The resolution grid targets consisted of a $12.5 \mu \mathrm{m}$ Ti backlighter foil attached at a right angle to a wire grid as shown in figure 3 . The wire grid had alternating 16 and $32 \mu \mathrm{m}$ copper wires with 130 $\mu \mathrm{m}$ center-to-center spacings. The spectrometer was focused on the grid so that it viewed the grid 


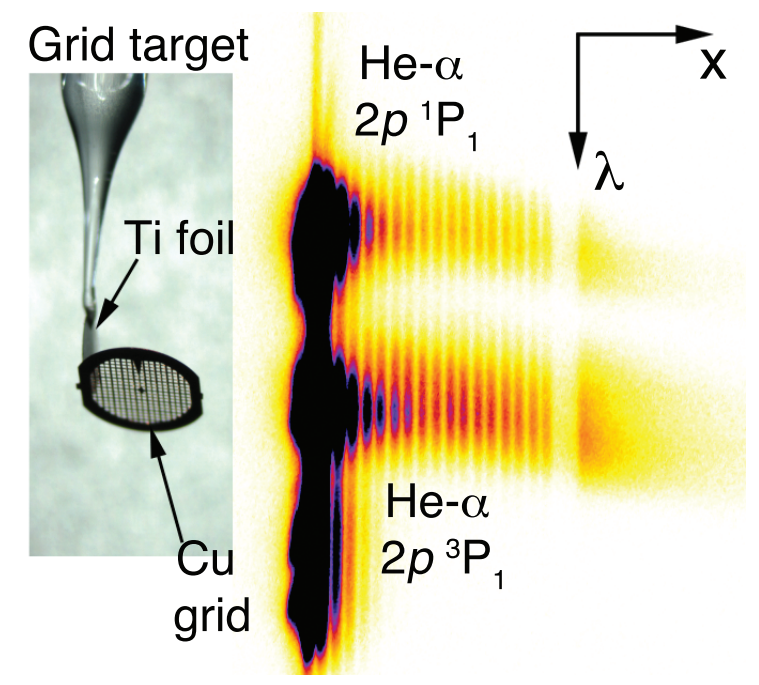

Figure 3. A resolution grid target and the resulting spatio-spectrograph from the imaging spectrometer. The image is resolved spatially along the horizontal axis and spectrally along the vertical. The Ti foil produces the saturated vertical line on the left while the spatial modulation in brightness from the grid is visible in the two helium-like emission lines. The full spectral range, spanning $350 \mathrm{eV}$ centered at $4680 \mathrm{eV}$, is not shown.

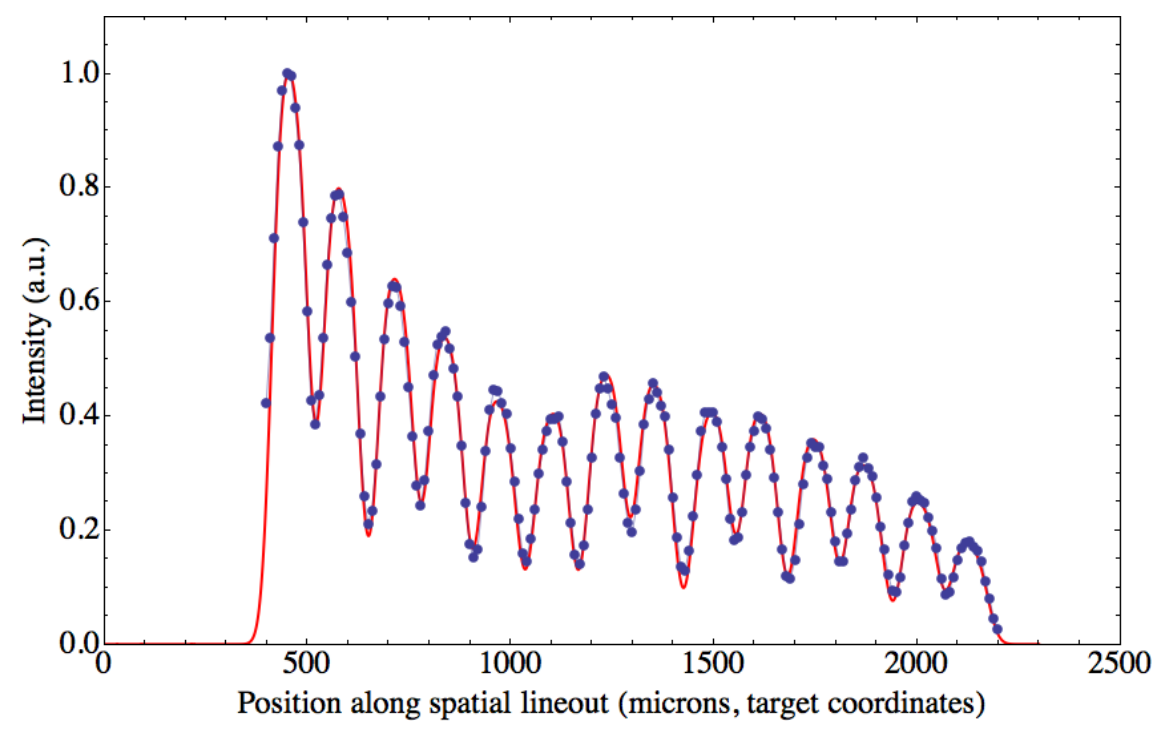

Figure 4. Plotted is a spatial lineout through the $2 p^{3} \mathrm{P}_{1}$ line in figure 3 (purple dots) and the fit to the data (red line). The FWHM resolution of the image is $48 \mu \mathrm{m}$

face-on. The foils were irradiated using $200 \mathrm{~J}$ of $527 \mathrm{~nm}$ laser light in $1.2 \mathrm{~ns}$ pulses. X-rays created within the extended plasma plume were spatially modulated through the grid and were imaged by the crystal onto a BAS-SR image plate.

A spatio-spectrograph from a resolution grid shot is shown in figure 3. All 18 grid spacings as well as the right edge of the rim of the grid are visible in the image, corresponding to a field of view of at least $3 \mathrm{~mm}$. The spectral direction of the image shows the two prominent He- $\alpha$ lines in the Ti emission as well as weaker transitions at lower energies. 


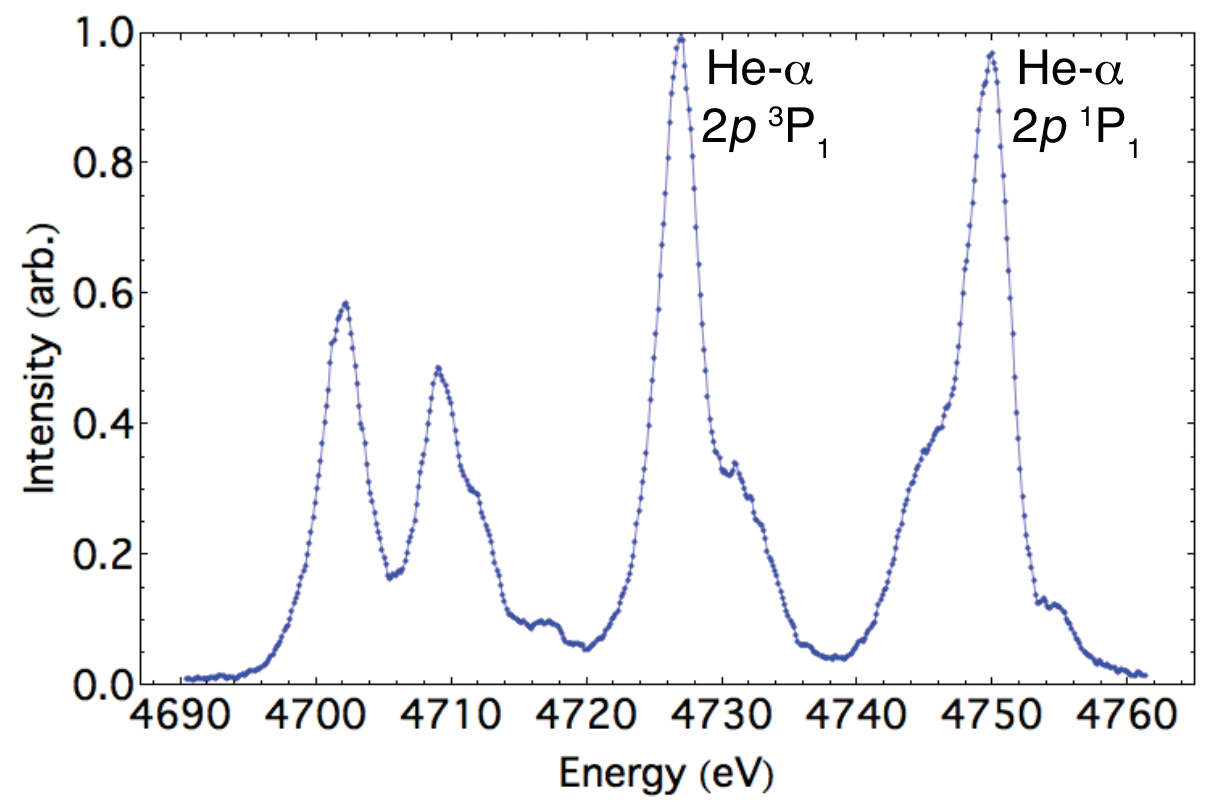

Figure 5. Plotted is a spectral lineout left of the foil in figure 3. The FWHM spectral resolution is $4 \mathrm{eV}$.

The spatial resolution was estimated by fitting a simulated image to the spatial data in the image. The image plates were scanned at $25 \mu \mathrm{m}$ per pixel and the spatial magnification of the spectrometer was 2.5. From the Nyquist theorem, we would expect the lower-limit on the spatial resolution to be $20 \mu \mathrm{m}$. A spectrally integrated, spatial lineout of the unsaturated region in figure 3 is shown by the purple dots in figure 4. Given the known thickness and spacing of the grid wires, we can fit the spatial lineout to estimate the spatial width of the point spread function (PSF). An idealized, one dimensional profile that represents the x-ray transmission through the grid was convolved with a Gaussian PSF. The width of the PSF was varied over a range of values to minimize the residuals of the resulting fit. The best fit indicates that the FWHM of the PSF $48 \mu \mathrm{m}$.

The demonstrated spatial resolution of the spectrometer is believed to be dominated by broadening from within the image plate detector. The image plates used in the experiments were Fuji BAS-SR scanned with a FLA-7000. The edge response function of the image plate was measured by placing a knife edge in front of the detector. The derivative of the profile was fitted to a Gaussian, yielding a width of $105 \mu \mathrm{m}$ on the detector plane. This value is consistent with those reported by Seely et al. [39]. We assume that the resolution of the images is the quadrature sum of the intrinsic crystal resolution and the spreading from the image plate. With the images resolved to $48 \mu \mathrm{m}$ and image plate broadening of $105 \mu \mathrm{m} /(M=2.5)=42 \mu \mathrm{m}$, the intrinsic crystal resolution was then $<25 \mu \mathrm{m}$. Future experiments plan to use deep-depletion x-ray CCDs as the detector, where higher resolution is expected since the spatial broadening due to charge sharing is only on the order of a few $\mu \mathrm{m}$ [40].

The demonstrated spatial resolution of the spectrometer is believed to be at the low-end of what is possible. The limits on the spatial resolution may be a consequence of two effects. The first is the fact that the toroidal crystal spectrometers lack the rotational symmetry of spherical crystals and such must be precisely aligned over six axes [41]. Subtle errors in the positioning 

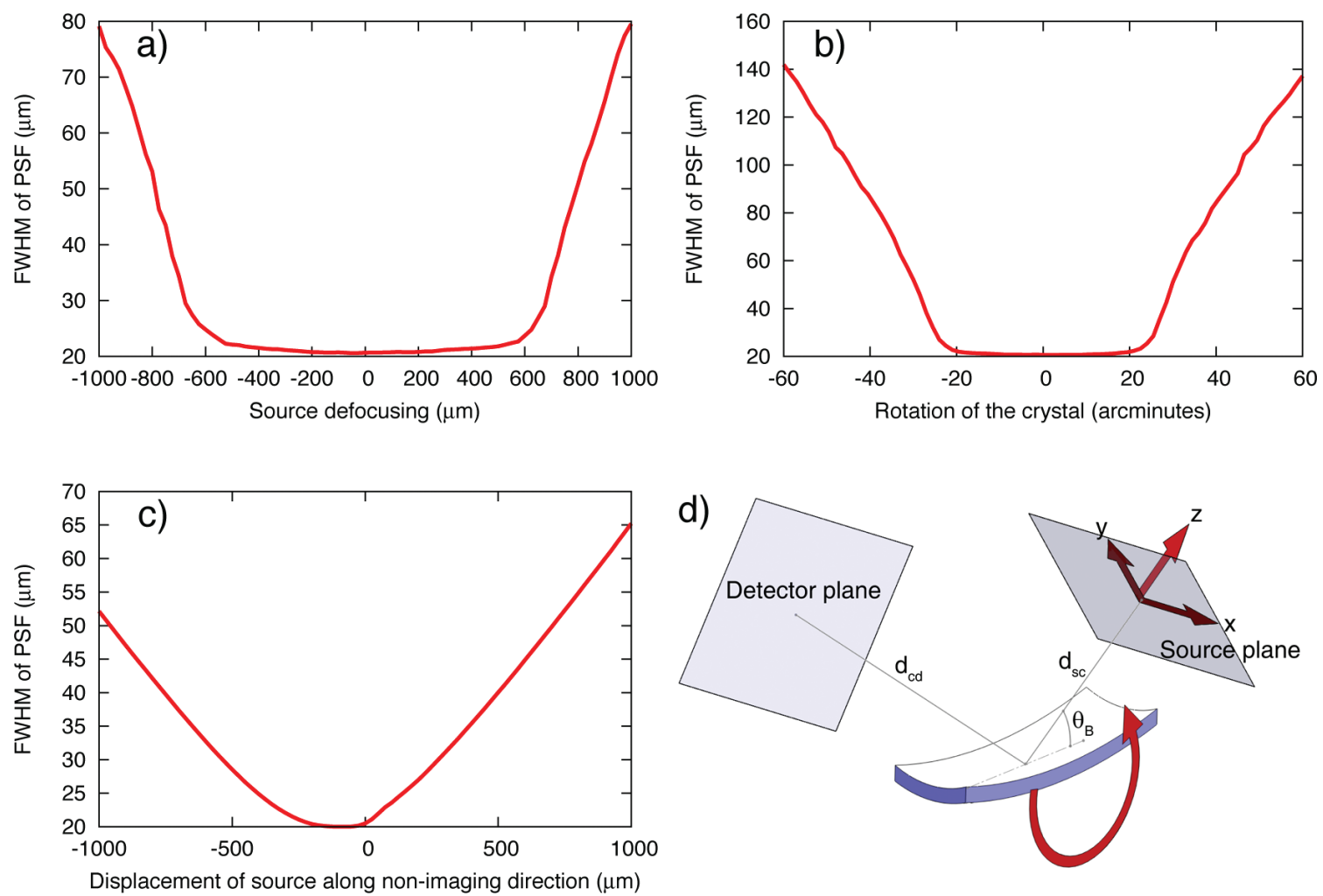

Figure 6. These plots summarize the results of the ray tracing analysis to assess defocusing errors. The coordinate system used in the ray tracing is shown in part d). The ordinate in these plots is the FWHM of the PSF divided by the magnification, to yield the PSF width in source coordinates. This value is summed in quadrature with the Nyquist-limited resolution of $20 \mu \mathrm{m}$. In plot a), a point source initially at the position of best focus was displaced along the z-axis to evaluate the effect of defocusing through the source-to-crystal vector. For part b), the crystal was rotated about the imaging $\mathrm{x}$-axis to change the angle of incidence off of the Bragg angle. Plot c) shows the results of displacing the point source along the non-imaging $y$-axis to quantify source broadening.

of the source and the setting of the Bragg angle can degrade the spatial resolution by defocusing errors. The second effect comes from the broadening of the source perpendicular to the source-tocrystal vector and imaging axis. The image becomes defocused as the spatial resolution elements move off of the imaging axis.

Tests were also done to determine the depth of field of the spectrometer. The source was stepped over a distance of $\pm 250 \mu \mathrm{m}$ along the source-to-crystal vector. There was little significant systematic change in the spatial resolution, only fluctuations of $\pm 5 \mu \mathrm{m}$. This indicates that the spectrometer has a depth of field of at least $0.5 \mathrm{~mm}$.

To assess the effect of positioning errors on the spatial resolution, a ray tracing analysis was performed on the spectrometer configuration fielded on Trident for a variety of positioning errors. The results are summarized in figure 6. The three largest defocusing effects were found to be source defocusing, rotation of the crystal affecting the Bragg angle, and lengthening of the spatial resolution elements in the non-imaging direction. The demonstrated spatial resolution is likely a combination of these three effects, which will have to be more tightly controlled in future exper- 
iments. Other positioning errors, including detector defocusing and displacements of the source along the imaging axis, were found to have little impact on the spatial resolution.

The depth of field along the source-to-crystal vector was found to be $\pm 600 \mu \mathrm{m}$. As our experimental tests showed little variation in the spatial resolution over $500 \mu \mathrm{m}$, it is likely that this distance was correctly set in our experiments. The spatial resolution rapidly worsens for angular displacements greater than 20 arcminutes, which may be the ultimate culprit. Displacing the source along the non-imaging $y$-axis has a similar effect on the spatial resolution as the rotational errors. The plume was allowed to freely expand as it shone through the resolution grid. The dimensions of the source in the non-imaging axis are unknown, but the uniform spatial resolution across the grid suggests that the plume was smaller than the $3 \mathrm{~mm}$ grid.

It is essential for future applications of the imaging spectrometer to XRTS experiments to limit the extent of the scattering volume along the non-imaging dimension. Extending the length of the spatial resolution elements will integrate over more signal and yield a brighter signal. However, the added signal from the edges will contribute less and less to central peak of the PSF and instead form a broad foot which contributes little to the contrast in the image

The spectral resolution was found to be unaffected by the extended source and positioning errors. Fitting a Gaussian to the two main peaks in the spectrum shown in figure 5 yields a $4 \mathrm{eV}$ FWHM resolution. This resolution is sufficient to see multiple lines around the main heliumlike transitions. For the $\mathrm{He}-\alpha$ line at $4750 \mathrm{eV}$, the integrated reflectivity was calculated using the XOP simulation code [42] for unpolarized light as $0.130 \mathrm{mrad}$. Using (2.17), we can calculate the geometrically modified integrated reflectivity. The spectral resolution due to geometric effects can be estimated as simply

$$
\Delta E=\frac{h c}{2 d}\left(\frac{1}{\sin \left(\theta_{B}\right)}-\frac{1}{\sin \left(\theta_{b}-R_{\mathrm{int}}^{\prime}\right)}\right)
$$

This expression gives a value for the spectral resolution of $\Delta E=4.3 \mathrm{eV}$ at $4750 \mathrm{eV}$. This value is a bit higher than the experimentally demonstrated resolution, likely because of errors in the calculated value for the integrated reflectivity.

In principle, it is possible to design an instrument of this nature for any desired x-ray source. For a given transition energy, the limited number of crystal planes that yield high integrated reflectivities constrains the choice of the Bragg angle. Higher x-ray energies generally result in lower Bragg angles and reduced spatial resolution from increased coma.

\section{Conclusion}

X-ray imaging spectroscopy using toroidally-curved crystals offers a new diagnostic possibility to measure scattered radiation from dense plasmas. The diagnostic combines high spectral and spatial resolutions with a large field-of-view in space and wavelength. We have demonstrated an instrument which uses a Ge crystal set to the He- $\alpha$ lines of titanium with spectral resolution of $\frac{E}{\Delta E}$ $=1200$, a spectral range of $350 \mathrm{eV},<25 \mu \mathrm{m}$ spatial resolution, and a spatial range of at least $3 \mathrm{~mm}$.

A unique strength of the toroidal crystal is the lack of astigmatism which allows for the use of low Bragg angles. In this way, the toroidal crystal imaging spectrometer may easily be adapted to experiments on large high-energy density facilities where space in the target chamber is at a premium. 


\section{Acknowledgments}

The authors would like to thank the staff at the Trident Laser Facility and S.H. Glenzer for his valuable input. This work is funded by the Predictive Sciences Academic Alliances Program in NNSA-ASC via grant DEFC52- 08NA28616, by the NNSA-DS and SC-OFES Joint Program in High-Energy-Density Laboratory Plasmas, grant number DE-FG52-09NA29548, and by the National Laser User Facility Program, grant number DE-FG52-09NA29034. This work performed under the auspices of the U.S. Department of Energy by Los Alamos National Laboratory under contract DE-AC52-06NA25396.

\section{References}

[1] H.H. Johann, Die erzeugung lichtstarker röntgenspektren mit hilfe von konkavkristallen, Zeit. Phis. 69 (1931) 185.

[2] T. Johansson, Über ein neuartiges, genau fokussierendes röntgenspektrometer, Z. Phys. 82 (1933) 507.

[3] L. von Hámos, Formation of true x-ray images by reflection on crystal mirrors, Z. Kristallogr. 101 (1939) 17.

[4] Y. Aglitskiy et al., High-resolution monochromatic x-ray imaging system based on spherically bent crystals, Appl. Opt. 37 (1998) 5253.

[5] Y. Aglitskiy et al., The use of a spherically curved crystal spectrometer for $x$-ray measurements on electron beam ion trap, Phys. Scripta 58 (1998) 178.

[6] A. Pak et al., X-ray line measurements with high efficiency Bragg crystals, Rev. Sci. Instrum. 75 (2004) 3747.

[7] M.K. Urry, G. Gregori, O.L. Landen, A. Pak and S.H. Glenzer, X-ray probe development for collective scattering measurements in dense plasmas, J. Quant. Spectrosc. Radiat. Transfer 99 (2006) 636.

[8] B. Yaakobi and A. Burek, Crystal diffraction systems for x-ray spectroscopy, imaging, and interferometry of laser fusion targets, IEEE J. Quantum Electron. 19 (1983) 1841.

[9] A. Ya. Faenov et al., High-resolution x-ray spectroscopy of a subpicosecond-laser-produced silicon plasma, Phys. Rev. A 51 (1995) 3529.

[10] T.A. Pikuz et al., High-resolution $x$-ray spectromicroscopy of fs laser-produced plasma by tunable, high-luminosity spherical crystal spectrometers with x-ray CCD or MCP, Proc. SPIE 4504 (2001) 12.

[11] P. Monot et al., High-sensitivity, portable, tunable imaging $x$-ray spectrometer, Nucl. Instrum. Meth. A 484 (2002) 299.

[12] D.B. Sinars et al., Plasma imaging and spectroscopy diagnostics developed on 100-500-kA pulsed power devices, Proc. IEEE 92 (2004) 1110.

[13] D.B. Sinars et al., Measurements of K-shell Ar spectra from z-pinch dynamic hohlraum experiments made using a focusing spectrometer with spatial resolution, J. Quant. Spectrosc. Radiat. Transfer 99 (2006) 595.

[14] T.A. Shelvenko et al., Use of spherically bent crystals to diagnose wire array z pinches, Rev. Sci. Instrum. 75 (2004) 3681. 
[15] B.K.F. Young et al., High-resolution $x$-ray spectrometer based on spherically bent crystals for investigations of femtosecond laser plasmas, Rev. Sci. Instrum. 69 (1998) 4049.

[16] D.B. Sinars, G.R. Bennett, D.F. Wenger, M.E. Cuneo, and J.L. Porter, Evaluation of bent-crystal $x$-ray backlighting and microscopy techniques for the Sandia Z machine, Appl. Opt. 42 (2003) 4059.

[17] T.R. Boehly et al., Initial performance results of the OMEGA laser system, Opt. Commun. 133 (1997) 495.

[18] E.I. Moses and C.R. Wuest, The National Ignition Facility: Laser performance and first experiments, Fusion Sci. Technol. 57 (2005) 314.

[19] E. Förster, K. Gabel and I. Uschmann, New crystal spectrograph designs and their application to plasma diagnostics (invited), Rev. Sci. Instrum. 63 (1992) 5012.

[20] M. Vollbrecht, O. Treichel, I. Uschmann, K. Gäbel, R. Lebert and E. Förster, Soft-x-ray imaging with toroidally curved thallium acid phthalate crystals in the water window, Appl. Opt. 37 (1998) 1803.

[21] E. Förster, K. Gabel and I. Uschmann, X-ray microscopy of laser-produced plasmas with the use of bent crystals, Laser Part. Beams 9 (1991) 135.

[22] I.M. Hall, N.C. Woolsey, O. Renner and I. Uschmann, High-resolution x-ray spectroscopy using toroidally bent crystals, Central Laser Facility Rutherford, Appleton Laboratory Annual Report (2003-2004) pages 47-48.

[23] A.H. Compton, A Quantum Theory of the Scattering of X-rays by Light Elements, Phys. Rev. 21 (1923) 483.

[24] O.L. Landen et al., Dense matter characterization by $x$-ray Thomson scattering, J. Quant. Spectrosc. Radiat. Transfer 71 (2001) 465.

[25] G. Gregori, S.H. Glenzer, W. Rozmus, R.W. Lee and O.L. Landen, Theoretical model of $x$-ray scattering as a dense matter probe, Phys. Rev. E 67 (2003) 026412.

[26] S.H. Glenzer, G. Gregori, R.W. Lee, F.J. Rogers, S.W. Pollaine and O.L. Landen, Demonstration of spectrally resolved x-ray scattering in dense plasmas, Phys. Rev. Lett. 90 (2003) 175002.

[27] S.H. Glenzer et al., Observations of plasmons in warm dense matter, Phys. Rev. Lett. 98 (2007) 065002.

[28] G. Gregori et al., Electronic structure measurements of dense plasmas, Phys. Plasmas 11 (2004) 2754.

[29] H. Sawada et al., Diagnosing direct-drive, shock-heated, and compressed plastic planar foils with noncollective spectrally resolved x-ray scattering, Phys. Plasmas 14 (2007) 122703.

[30] T. Döppner et al., X-ray Thomson scattering for measuring dense beryllium plasma collisionality, J. Phys. Conf. Ser. 244 (2010) 032044.

[31] A.L. Kritcher et al., Demonstration of $x$-ray Thomson scattering using picosecond k-alpha $x$-ray sources in the characterization of dense heated matter, Rev. Sci. Instrum. 79 (2008) 10E739.

[32] H.J. Lee et al., X-ray Thomson-scattering measurements of density and temperature in shock-compressed beryllium, Phys. Rev. Lett. 102 (2009) 115001.

[33] T. Missalla, I. Uschmann, E. Förster, G. Jenke and D. von der Linde, Monochromatic focusing of subpicosecond $x$-ray pulses in the keV range, Rev. Sci. Instrum. 70 (1999) 1288.

[34] W.H. Zachariasen, X-ray Diffraction in Crystals, John Wiley and Sons (1944). 
[35] I. Uschmann, E. Förster, K. Gäbel, G. Hölzer and M. Ensslen, X-ray reflection properties of elastically bent perfect crystals in Bragg geometry, J. Appl. Crystallogr. 26 (1993) 405.

[36] S.H. Glenzer and R. Redmer, X-ray Thomson scattering in high energy density plasmas, Rev. Mod. Phys. 81 (2009) 1625.

[37] C. Welnak, P. Anderson, M. Khan, S. Singh and F. Cerrina, Recent developments in SHADOW, Rev. Sci. Instrum. 63 (1992) 865.

[38] N.K. Moncur, R.P. Johnson, R.G. Watt and R.B. Gibson, Trident: a versatile high-power Nd:glass laser facility for inertial confinement fusion experiments, Appl. Opt. 34 (1995) 4274.

[39] J.F. Seely, G.E. Holland, L.T. Hudson and A. Henins, X-ray modulation transfer functions of photostimulable phosphor image plates and scanners, Appl. Opt. 47 (2008) 5753.

[40] F. Zamponi et al., Characterization of a deep depletion, back-illuminated charge-coupled device in the x-ray range, Rev. Sci. Instrum. 76 (2005) 116101.

[41] J.A. Koch et al., 4.5- and 8-keV emission and absorption $x$-ray imaging using spherically bent quartz 203 and 211 crystals (invited), Rev. Sci. Instrum. 74 (2003) 2130.

[42] M. Sanchez del Rio and R.J. Dejus, XOP: a multiplatform graphical user interface for synchrotron radiation spectral and optics calculations, Proc. SPIE 3152 (1997) 148. 